\title{
Perspectivas dos estudantes universitários sobre Educação Interprofissional
}

\author{
University students' perspectives on Interprofessional Education \\ Perspectivas de los estudiantes universitarios sobre la Educación Interprofesional
}

Recebido: 06/04/2021 | Revisado: 12/04/2021 | Aceito: 14/04/2021 | Publicado: 27/04/2021

Luis Felipe Castro Cardoso

ORCID: https://orcid.org/0000-0003-4549-1045

Universidade Federal do Maranhão, Brasil

E-mail: Castrofelipec@hotmail.com

Adryemerson Pena Forte Ferreira

ORCID: https://orcid.org/0000-0001-6423-9756

Universidade Federal do Maranhão, Brasil

E-mail: adryemerson.pena@gmail.com

Matheus Campos Silva

ORCID: https://orcid.org/0000-0001-5706-6981

Universidade Federal do Maranhão, Brasil

E-mail: matheuscampos777@gmail.com

Carlos Alberto de Melo Filho

ORCID: https://orcid.org/0000-0002-9877-8341

Universidade Federal do Maranhão, Brasil

E-mail: carlos.amf@discente.ufma.br

Sara Fiterman Lima

ORCID: https://orcid.org/0000-0003-0015-3413 Universidade Federal do Maranhão, Brasil E-mail: sara.fiterman@ufma.br

Mayara Cristina Pinto da Silva

ORCID: https://orcid.org/0000-0002-1868-6931 Universidade Federal do Maranhão, Brasil E-mail: Mayara.silva@ufma.br

João de Jesus Oliveira Junior

ORCID: https://orcid.org/0000-0002-3259-6131 Universidade Federal do Maranhão, Brasil E-mail: joaodejota@gmail.com

Amanda Namíbia Pereira Pasklan ORCID: https://orcid.org/0000-0001-7193-4861 Universidade Federal do Maranhão, Brasil E-mail: amanda.namibia@ufma.br

\begin{abstract}
Resumo
Esta pesquisa objetivou avaliar a perspectiva dos estudantes da saúde sobre educação interprofissional em uma universidade pública do estado do Maranhão. Foi aplicado um questionário com variáveis sociodemográficas e de perfil dos acadêmicos e o instrumento RIPLS (Readiness for Interprofessional Learning Scale) adaptado, de forma online, com 254 acadêmicos dos cursos da saúde. Para a análise da diferença estatística significante foi calculado o teste t de Student e Anova para a diferença de médias e Qui-quadrado para a concordância das assertivas isoladamente, considerando o nível de significância de 5\%, sendo analisado no programa Stata versão 14. Os resultados identificaram que há uma alta disponibilidade dos estudantes dos cursos da saúde para a aprendizagem interprofissional, sendo mais aceita nos períodos clínicos/fase intermediária dos cursos, onde se sugere que as práticas colaborativas serão mais exigidas. Destaca-se que a maioria dos pesquisados se encontram em Zona de Conforto em relação a Educação Interprofissional e Práticas Colaborativas no ambiente acadêmico. O estudo destaca que atividades extracurriculares são ferramentas indispensáveis no processo de ensino-aprendizagem da educação interprofissional e práticas colaborativas. Conclui-se a importância da criação e manutenção de ambientes que abordem a discussão dessa temática, para que a prática interprofissional colaborativa ocorra de forma abrangente entre todos os acadêmicos dos cursos da saúde.
\end{abstract}

Palavras-chave: Educação interprofissional; Práticas interdisciplinares; Pesquisa interdisciplinar.

\begin{abstract}
This research aimed to evaluate the perspective of health students on interprofessional education at a public university in the state of Maranhão. A questionnaire was applied with sociodemographic and profile variables of the students and the RIPLS (Readiness for Interprofessional Learning Scale) instrument adapted online with 254 students from the health courses. For the analysis of the statistically significant difference, Student's t test and Anova were calculated for the difference in means and Chi-square for the agreement of the statements alone, considering the significance level of 5\%,
\end{abstract}


being analyzed in the Stata version 14 program. results identified that there is a high availability of students of health courses for interprofessional learning, being more accepted in clinical periods / intermediate phase of courses, where it is suggested that collaborative practices will be more required. It is noteworthy that the majority of those surveyed are in a Comfort Zone in relation to Interprofessional Education and Collaborative Practices in the academic environment. The study highlights that extracurricular activities are indispensable tools in the teaching-learning process of interprofessional education and collaborative practices. It concludes the importance of creating and maintaining environments that address the discussion of this theme, so that the collaborative interprofessional practice occurs comprehensively among all students of health courses.

Keywords: Interprofessional education; Interdisciplinary placement; Interdisciplinary research.

\section{Resumen}

Esta investigación tuvo como objetivo evaluar la perspectiva de los estudiantes de salud sobre la educación interprofesional en una universidad pública del estado de Maranhão. Se aplicó un cuestionario con variables sociodemográficas y de perfil de los estudiantes y el instrumento RIPLS (Readiness for Interprofessional Learning Scale) adaptado en línea con 254 estudiantes de los cursos de salud. Para el análisis de la diferencia estadísticamente significativa, se calcularon las pruebas t de Student y Anova para la diferencia de medias y Chi-cuadrado para la concordancia de los enunciados solo, considerando el nivel de significancia del 5\%, que se analiza en el programa Stata versión 14. Los resultados identificaron que existe una alta disponibilidad de estudiantes de cursos de salud para el aprendizaje interprofesional, siendo más aceptados en períodos clínicos / fase intermedia de cursos, donde se sugiere que las prácticas colaborativas serán más requeridas. Es de destacar que la mayoría de los encuestados se encuentran en una Zona de Confort en relación a la Educación Interprofesional y las Prácticas Colaborativas en el ámbito académico. El estudio destaca que las actividades extraescolares son herramientas indispensables en el proceso de enseñanzaaprendizaje de la educación interprofesional y las prácticas colaborativas. Se concluye la importancia de crear y mantener ambientes que aborden la discusión de este tema, para que la práctica colaborativa interprofesional se produzca de manera integral entre todos los estudiantes de los cursos de salud.

Palabras clave: Educación interprofesional; Prácticas interdisciplinarias; Investigación interdisciplinaria.

\section{Introdução}

Importantes desafios para o trabalho e formação em saúde se destacam no cenário atual. Novos perfis demográficos, epidemiológicos, e riscos infeciosos, ambientais e comportamentais, trazem a tona a necessidade de se repensar o atual processo de trabalho em saúde, pois os problemas e necessidades enfrentados são cada vez mais complexos, e exigem mudanças de paradigmas em relação a educação e assistência em saúde (Crisp \& Chen, 2014; Frenk et al., 2010). Dentre tais estratégias, a educação interprofissional (EIP), vem sendo reconhecida como a principal técnica para formar profissionais de saúde preparados para o TE (Institute of Medicine, 2015; Organização Mundial de Saúde, 2010).

Por sua vez, no contexto universitário, a EIP encontra diferentes obstáculos. Os cursos da saúde, funcionam em estruturas próprias, dificultando assim a interação entre eles, e a formação específica é muito forte, influenciando na construção das identidades profissionais. O processo de formação é ainda muito conteudista, dificultando estratégias para a formação de atitudes, habilidades e valores pautados na colaboração e no trabalho em equipe. Nesse contexto, formar profissionais de saúde dispostos e aptos ao trabalho conjunto num contexto institucional onde prevalece a formação em separado, se transforma em grande desafio (Costa, 2016).

A Organização Mundial de Saúde publicou em 2010, o Marco para ação em educação interprofissional e prática colaborativa, onde reconhece que existem evidências suficientes para afirmar que a educação interprofissional (EIP) prepara os profissionais para o enfrentamento das complexas demandas atuais da população (Organização Mundial de Saúde, 2010). Existe uma demanda no trabalho em saúde que transcende os fazeres individualizados de cada profissão, sendo necessário fortalecer a compreensão de que as especificidades são complementares, e que no TE o profissional não abre mão da sua especificidade, mas valoriza o trabalho cooperativo para o atendimento das complexas e dinâmicas necessidades sociais e de saúde, atribuindo-lhes centralidade (Costa, 2016; Casanova et al., 2018).

Nesse contexto, diversos organismos internacionais vêm estimulando cada vez mais estratégias de formação em saúde capazes de desenvolver competências para o efetivo trabalho em equipe (TE) (Organização Mundial de Saúde, 2010). 
Destaca-se que o efetivo TE se dá quando profissionais de diferentes áreas da saúde compartilham uma identidade de equipe, com clareza dos papéis de cada membro, objetivos adequadamente traçados, e atividades realizadas de forma integrada e interdependentes (Reeves, Xyrichis \& Zwarenstein, 2018). Além disso, Reeves (2010) destaca a necessidade de franca comunicação, respeito mútuo, encontros regulares e esforços contínuos para manter a união da equipe.

A EIP, além de potencializar o TE, ampara e promove a prática colaborativa, entendendo-a como a tomada de decisão compartilhada e construída a partir da aliança de saberes entre profissionais diversos por meio do diálogo (Organização Mundial de Saúde, 2010). Adicionalmente, a EIP viabiliza ambientes onde o estudante pode fortalecer a sua identidade profissional pelo envolvimento com estudantes de outras profissões, permitindo a evolução de grupos de trabalho para equipes de trabalho aptas a oferecerem bons serviços holísticos (Haugland et al, 2019).

A colaboração interprofissional (CI), é uma estratégia que possibilita uma práxis participativa entre os membros de uma equipe, permitindo a introdução de novas práticas assistenciais e diferentes visões sobre o relacionamento entre profissionais de saúde de diferentes formações, e entre estes profissionais e seus pacientes (Barros \& Ellery, 2016).

A relevância deste estudo justifica-se pela importância cada vez maior de inserção da prática interprofissional e da prática colaborativa na formação da força de trabalho para assistência em saúde. Dessa forma, pesquisas relacionadas a prática interprofissional com foco graduação em área saúde, podem ajudar na compreensão das relações interprofissionais nos serviços de saúde, instrumentalizando estratégias para melhoria do processo de ensino aprendizagem nas universidades brasileiras principalmente no que tange à integralidade, às mudanças necessárias ao modelo técnico-assistencial.

Nesse cenário, o presente estudo teve como o objetivo de avaliar a perspectiva dos estudantes da saúde sobre educação interprofissional em uma universidade pública do estado do Maranhão.

\section{Metodologia}

Esta pesquisa foi realizada por aplicação de questionário online com acadêmicos dos cursos da saúde de um campus da Universidade Federal do Maranhão, no período de maio a junho de 2020.

A amostra foi por conveniência, na qual foram convidados a participar todos os acadêmicos matriculados em algum curso da saúde. O estudo teve como critério de inclusão: estar regularmente matriculado no curso, e ter acesso a internet e a meio eletrônico, como smartphone, tablet ou computador. Foram excluídos os acadêmicos que trancaram o curso no período da coleta dos dados, e que estavam de licença maternidade ou de saúde.

Os cursos da saúde do campus possuem regularmente matriculados: 117 acadêmicos no curso de Educação Física, 197 acadêmicos no curso de Enfermagem, e 334 acadêmicos no curso de Medicina.

A amostra foi calculada de forma que houvesse $95 \%$ de grau de confiança e 5\% de margem de erro, totalizando a serem entrevistados 242 acadêmicos.

Utilizou-se um questionário com variáveis sociodemográficas e de perfil dos acadêmicos, no qual foram analisados: idade, sexo, estado civil, atividade religiosa, participação em atividades extracurriculares, período da graduação, curso de graduação, e formação anterior de nível superior.

Também foi aplicado o instrumento RIPLS (Readiness for Interprofessional Learning Scale) adaptado, o qual contém 26 itens que envolvem perguntas sobre trabalho em equipe e colaboração (TEC), identidade profissional (IP) e atenção centrada no paciente (ACP), avaliados por uma escala Likert de cinco categorias, com a seguinte pontuação: (1) discordo totalmente; (2) discordo; (3) não concordo nem discordo; (4) concordo; (5) concordo totalmente (Nuto et al., 2017).

O questionário foi hospedado no Google Forms e foi enviado aos participantes o link contendo o Termo de Consentimento Livre e Esclarecido, o questionário, e a escala a serem respondidos. 
Os dados coletados foram organizados na forma de banco de dados em planilhas do programa Excel na forma de percentuais. Foram organizados em três grupos referentes a cada um dos cursos da área da saúde do campus: Enfermagem, Educação física e Medicina o que permitiu realizar comparações entre os diferentes resultados. O banco de dados foi exportado para o programa Stata 14.0 onde se procedeu a análise dos dados obtidos. Para a análise da diferença estatística significante foi calculado o teste $\mathrm{t}$ de Student e Anova para a diferença de médias e Qui-quadrado para a concordância das assertivas isoladamente, considerando o nível de significância de 5\%. Os resultados estão apresentados no formato de tabelas.

A confidencialidade dos dados coletados será mantida e respeitadas as diretrizes da Resolução no 466/12 do Conselho Nacional de Saúde. O presente estudo foi submetido ao comitê de ética da Universidade Federal do Maranhão obtendo aprovação, com certificado de número 29652520.0.0000.5087.

\section{Resultados}

Participaram deste estudo 254 estudantes, sendo apresentados na Tabela 1 sobre as características sociodemográficas e perfil acadêmico. 
Tabela 1 - Frequência e proporção das características sociodemográficas e acadêmicas dos participantes da pesquisa. PinheiroMA, 2020

\begin{tabular}{|c|c|c|}
\hline Características & $\mathbf{n}$ & $\%$ \\
\hline \multicolumn{3}{|l|}{ Sexo } \\
\hline Feminino & 159 & 62,6 \\
\hline Masculino & 95 & 37,4 \\
\hline \multicolumn{3}{|l|}{ Faixa Etária (anos) } \\
\hline 16 a 20 & 60 & 23,62 \\
\hline 21 a 25 & 123 & 48,43 \\
\hline 26 a 30 & 45 & 17,72 \\
\hline 31 ou mais & 26 & 10,24 \\
\hline \multicolumn{3}{|l|}{ Estado Civil } \\
\hline Solteiro & 215 & 84,65 \\
\hline Casado/União Estável & 36 & 14,17 \\
\hline Separado/Divorciado & 3 & 1,18 \\
\hline \multicolumn{3}{|l|}{ Atividade Religiosa } \\
\hline Nenhuma & 21 & 8,27 \\
\hline Católico & 148 & 58,27 \\
\hline Evangélico & 64 & 25,20 \\
\hline Espírita & 3 & 1,18 \\
\hline Outros & 5 & 1,97 \\
\hline Agnóstico & 9 & 3,54 \\
\hline Ateu & 4 & 1,57 \\
\hline \multicolumn{3}{|l|}{ Participa de Atividade Extra } \\
\hline Sim & 152 & 59,84 \\
\hline Não & 102 & 40,16 \\
\hline \multicolumn{3}{|l|}{ Qual atividade } \\
\hline Nenhuma & 103 & 40,55 \\
\hline PET-Saúde & 32 & 12,60 \\
\hline Iniciação científica & 28 & 11,02 \\
\hline Liga acadêmica & 104 & 40,94 \\
\hline Projeto de extensão & 54 & 21,26 \\
\hline Outros & 15 & 5,91 \\
\hline \multicolumn{3}{|l|}{ Período da Graduação } \\
\hline 1 ao $3^{\circ}$ & 84 & 33,07 \\
\hline $4^{\circ}$ ao $7^{\circ}$ & 78 & 30,71 \\
\hline A partir do $8^{\circ}$ & 92 & 36,22 \\
\hline \multicolumn{3}{|l|}{ Curso da Graduação } \\
\hline Educação Física & 64 & 25,2 \\
\hline Enfermagem & 87 & 34,25 \\
\hline Medicina & 103 & 40,55 \\
\hline \multicolumn{3}{|l|}{ Possui outra graduação } \\
\hline Sim & 39 & 15,35 \\
\hline Não & 215 & 84,65 \\
\hline \multicolumn{3}{|l|}{ Qual a segunda graduação } \\
\hline Área da saúde & 25 & 8,84 \\
\hline Fora da área da saúde & 14 & 5,51 \\
\hline Não possui & 215 & 84,65 \\
\hline
\end{tabular}

Fonte: Autores.

A maioria dos entrevistados foi composta por mulheres (62,6\%), com idade na faixa etária entre 21 a 25 anos $(48,43 \%)$, estado civil solteiro $(84,65 \%)$, atividade religiosa catolicismo $(58,27 \%)$. A maioria encontrava-se nos períodos finais do curso 
(36,22\%), estando na primeira graduação $(84,65 \%)$, e participando de alguma atividade extra $(59,84 \%)$, sendo a atividade mais relatada a de liga acadêmica $(40,94 \%)$ (Tabela 1$)$.

Na Tabela 2, que descreve a média dos fatores 1, 2 e 3 da escala de RIPLS segundo características sociodemográficas e perfil acadêmico dos entrevistados, o estudo evidenciou que não houve diferença significativa entre os sexos $(\mathrm{p}$-valor $=0.524)$ em desenvolver as competências colaborativas.

Tabela 2 - Média dos fatores da escala de RIPLS segundo características sociodemográficas e acadêmicas dos participantes da pesquisa. Pinheiro-MA, 2020.

\begin{tabular}{|c|c|c|c|c|c|c|c|c|}
\hline \multirow{2}{*}{ Variáveis } & \multicolumn{2}{|c|}{ Fator 1} & \multicolumn{2}{|c|}{ Fator 2} & \multicolumn{2}{|c|}{ Fator 3} & \multicolumn{2}{|c|}{ Total } \\
\hline & Média & p-valor & Média & p-valor & Média & p-valor & Média & p-valor \\
\hline \multicolumn{9}{|l|}{ Sexo } \\
\hline Feminino & 61,93 & \multirow{2}{*}{0.878} & 27,38 & \multirow{2}{*}{0.02} & 21,88 & \multirow{2}{*}{0.096} & 111,19 & \multirow{2}{*}{0.524} \\
\hline Masculino & 59,01 & & 26,17 & & 21,20 & & 106,38 & \\
\hline \multicolumn{9}{|l|}{ Faixa Etária (anos) } \\
\hline 16 a 20 & 59,31 & \multirow{4}{*}{0.176} & 27,6 & \multirow{4}{*}{0.732} & 21,53 & \multirow{4}{*}{0.326} & 108,45 & \multirow{4}{*}{0.362} \\
\hline 21 a 25 & 61,60 & & 26,78 & & 21,71 & & 110,10 & \\
\hline 26 a 30 & 60,75 & & 26,95 & & 21,75 & & 109,46 & \\
\hline 31 ou mais & 60,84 & & 26,07 & & 21,19 & & 108,11 & \\
\hline \multicolumn{9}{|l|}{ Estado Civil } \\
\hline Solteiro & 60,77 & \multirow{3}{*}{0.666} & 27,05 & \multirow{3}{*}{0.462} & 21,60 & & 109,43 & \multirow{3}{*}{0.871} \\
\hline Casado/União Estável & 61,38 & & 26,36 & & 21,97 & 0.279 & 109,72 & \\
\hline Separado/Divorciado & 58,66 & & 25,33 & & 18,66 & & 102,66 & \\
\hline \multicolumn{9}{|l|}{ Atividade Religiosa } \\
\hline Nenhuma & 60,19 & \multirow{7}{*}{0.933} & 27,04 & \multirow{7}{*}{0.712} & 21,76 & & 109,00 & \multirow{7}{*}{0.951} \\
\hline Católico & 61,79 & & 27,02 & & 21,74 & & 110,56 & \\
\hline Evangélico & 60,18 & & 26,59 & & 21,37 & & 108,15 & \\
\hline Espírita & 61,00 & & 28,00 & & 22,00 & 0.727 & 111,00 & \\
\hline Outros & 53,00 & & 24,60 & & 21,20 & & 98,80 & \\
\hline Agnóstico & 54,33 & & 28,00 & & 20,88 & & 103,22 & \\
\hline Ateu & 63,50 & & 28,00 & & 22,50 & & 114,00 & \\
\hline \multicolumn{9}{|c|}{ Participa de Atividade Extra } \\
\hline Sim & 62,03 & \multirow{2}{*}{0.152} & 26,86 & 0282 & 21,88 & 0732 & 110,78 & 0511 \\
\hline Não & 59,05 & & 27,03 & 0.282 & 21,23 & 0.132 & 107,33 & 0.511 \\
\hline Período da Graduação & & & & & & & & \\
\hline 1 ao $3^{\circ}$ & 61,20 & & 27,03 & & 22,04 & & 110,28 & \\
\hline $4^{\circ}$ ao $7^{\circ}$ & 59,78 & 0.104 & 27,26 & 0.032 & 21,21 & 0.319 & 108,26 & 0.513 \\
\hline A partir do $8^{\circ}$ & 61,40 & & 26,55 & & 21,58 & & 109,54 & \\
\hline Curso da Graduação & & & & & & & & \\
\hline Educação Física & 58,00 & & 26,45 & & 20,59 & & 105,04 & \\
\hline Enfermagem & 61,25 & 0.264 & 27,39 & 0.752 & 21,57 & 0.692 & 110,21 & 0.797 \\
\hline Medicina & 62,25 & & 26,84 & & 22,31 & & 111,40 & \\
\hline Possui outra graduação & & & & & & & & \\
\hline Sim & 60,23 & 0875 & 26,33 & 0686 & 21,53 & 0773 & 108,10 & 0620 \\
\hline Não & 60,94 & 0.875 & 27,04 & 0.080 & 21,64 & $0.7 / 3$ & 109,63 & 0.020 \\
\hline Qual a segunda graduas & & & & & & & & \\
\hline Área da saúde & 61,40 & & 26,88 & & 22,08 & & 110,36 & \\
\hline Fora da área da saúde & 58,14 & 0.722 & 25,35 & 0.476 & 20,57 & 0.951 & 104,07 & 0.861 \\
\hline Não possui & 60,94 & & 27,04 & & 21,64 & & 109,63 & \\
\hline
\end{tabular}

*ANOVA e CHI2. Fonte: Autores. 
Quando avaliamos isoladamente o Fator 2 (F2), que diz respeito a Identidade Profissional (IP), este encontra-se mais consolidado entre as mulheres $(27,38 ;$ p-valor $=0.02)$. O Fator $1(F 1)-$ Trabalho em Equipe e Colaboração (TEC) - e o Fator 3 (F3) - Atenção Centrada no Paciente - não apresentaram significância estatística entre os sexos (Tabela 2).

A média da faixa etária predominante para o desenvolvimento das competências colaborativas foi de 110,10, sendo os mais velhos apresentando menor score quando comparado aos demais $(108,11)$. Porém, sem significância forte entre as faixas etárias (Tabela 2).

Quanto aos 152 acadêmicos que disseram fazer alguma atividade extracurricular, estes apresentaram maior disponibilidade para aprendizagem interprofissional $(110,78)$ quando comparados com aqueles que não participavam de nenhuma atividade $(107,33)$ (Tabela 2).

Quando se avaliou as potencialidades dos estudantes em relação a IP, aqueles que se encontravam no ciclo clínico/fase intermediária ( $4^{\circ}$ ao $7^{\circ}$ período) apresentaram os maiores scores $(27,26$; p-valor $=0,032)$. Em contrapartida, evidenciou-se que os alunos que estavam na fase final do curso eram os que tinham menos prontidão para essa competência (Tabela 2).

Os acadêmicos de Medicina $(110,40)$ foram os que apresentaram maior potencial para a colaboração interprofissional, seguido pelos da Enfermagem $(110,21)$ e depois os da Educação Física $(105,04)$. Somente no F2, o curso da Enfermagem $(27,39)$ apresentou scores maiores do que da Medicina $(26,84)$ (Tabela 2).

Os alunos que relataram estar na primeira graduação somaram 109,63 no score de prontidão para aprendizagem interprofissional, já aqueles em segunda graduação pontuaram 109, 63, porém sem significância estatística forte (0.620) (Tabela 2).

A Tabela 3 apresenta a frequência que os fatores 1, 2 e 3 da escala de RIPLS de acordo com as características sociodemográficas e perfil acadêmico dos entrevistados. 
Tabela 3 - Frequência dos fatores da escala de RIPLS segundo características sociodemográficas e acadêmicas dos participantes da pesquisa. Pinheiro-MA, 2020.

\begin{tabular}{|c|c|c|c|c|c|c|c|c|c|c|c|c|}
\hline \multirow{2}{*}{ Variáveis } & \multicolumn{4}{|c|}{ Fator 1} & \multicolumn{4}{|c|}{ Fator 2} & \multicolumn{4}{|c|}{ Fator 3} \\
\hline & $\mathrm{ZC}^{1}$ & $\mathbf{Z A}^{\mathbf{2}}$ & $\mathbf{Z P}^{\mathbf{3}}$ & p-valor & $\mathbf{Z C}$ & $\mathbf{Z A}$ & $\mathbf{Z P}$ & p-valor & $\mathbf{Z C}$ & $\mathbf{Z A}$ & $\mathbf{Z P}$ & $p$-valor \\
\hline \multicolumn{13}{|l|}{ Sexo } \\
\hline Feminino & 153 & 5 & 1 & \multirow{2}{*}{0.005} & 125 & 33 & 1 & \multirow{2}{*}{0.016} & 149 & 10 & 0 & \multirow{2}{*}{0.047} \\
\hline Masculino & 82 & 13 & 0 & & 60 & 35 & 0 & & 82 & 13 & 0 & \\
\hline \multicolumn{13}{|l|}{ Faixa Etária (anos) } \\
\hline 16 a 20 & 53 & 7 & 0 & \multirow{4}{*}{0.712} & 47 & 13 & 0 & \multirow{4}{*}{0.076} & 58 & 2 & 0 & \multirow{4}{*}{0.054} \\
\hline 21 a 25 & 115 & 7 & 1 & & 91 & 32 & 0 & & 109 & 14 & 0 & \\
\hline 26 a 30 & 42 & 3 & 0 & & 29 & 16 & 0 & & 43 & 2 & 0 & \\
\hline 31 ou mais & 25 & 1 & 0 & & 18 & 7 & 1 & & 21 & 5 & 0 & \\
\hline \multicolumn{13}{|l|}{ Estado Civil } \\
\hline Solteiro & 200 & 14 & 1 & \multirow{3}{*}{0.478} & 159 & 56 & 0 & \multirow{3}{*}{0.071} & 197 & 18 & 0 & \multirow{3}{*}{0.002} \\
\hline Casado/União Estável & 33 & 3 & 0 & & 25 & 10 & 1 & & 33 & 3 & 0 & \\
\hline Separado/Divorciado & 2 & 1 & 0 & & 1 & 2 & 0 & & 1 & 2 & 0 & \\
\hline \multicolumn{13}{|l|}{ Atividade Religiosa } \\
\hline Nenhuma & 21 & 1 & 0 & \multirow{7}{*}{0.000} & 15 & 6 & 0 & \multirow{7}{*}{0.743} & 19 & 2 & 0 & \multirow{7}{*}{0.424} \\
\hline Católico & 141 & 7 & 0 & & 108 & 39 & 1 & & 138 & 10 & 0 & \\
\hline Evangélico & 59 & 4 & 1 & & 49 & 15 & 0 & & 55 & 9 & 0 & \\
\hline Espírita & 3 & 0 & 0 & & 2 & 1 & 0 & & 3 & 0 & 0 & \\
\hline Outros & 2 & 3 & 0 & & 1 & 4 & 0 & & 5 & 0 & 0 & \\
\hline Agnóstico & 6 & 3 & 0 & & 7 & 2 & 0 & & 7 & 2 & 0 & \\
\hline Ateu & 4 & 0 & 0 & & 3 & 1 & 0 & & 4 & 0 & 0 & \\
\hline \multicolumn{13}{|c|}{ Participa de Atividade Extra } \\
\hline Sim & 144 & 8 & 0 & \multirow{2}{*}{0.177} & 115 & 36 & 1 & \multirow{2}{*}{0.296} & 137 & 15 & 0 & 0581 \\
\hline Não & 91 & 10 & 1 & & 70 & 32 & 0 & & 94 & 8 & 0 & 0.501 \\
\hline Período da Graduação & & & & & & & & & & & & \\
\hline 1 ao $3^{\circ}$ & 76 & 8 & 0 & & 60 & 24 & 0 & & 81 & 3 & 0 & \\
\hline $4^{\circ}$ ao $7^{\circ}$ & 72 & 5 & 1 & 0.486 & 62 & 16 & 0 & 0.379 & 70 & 8 & 0 & 0.083 \\
\hline A partir do $8^{\circ}$ & 87 & 5 & 0 & & 63 & 28 & 1 & & 80 & 12 & 0 & \\
\hline Curso da Graduação & & & & & & & & & & & & \\
\hline Educação Física & 55 & 9 & 0 & & 42 & 22 & 0 & & 55 & 9 & 0 & \\
\hline Enfermagem & 84 & 2 & 1 & 0.048 & 70 & 16 & 1 & 0.136 & 80 & 7 & 0 & 0.260 \\
\hline Medicina & 96 & 7 & 0 & & 73 & 30 & 0 & & 96 & 7 & 0 & \\
\hline Possui outra graduação & & & & & & & & & & & & \\
\hline Sim & 35 & 4 & 0 & 0646 & 27 & 11 & 1 & $0 \Omega 60$ & 34 & 5 & 0 & ( 373 \\
\hline Não & 200 & 14 & 1 & 0.040 & 158 & 57 & 0 & 0.000 & 197 & 18 & 0 & 0.575 \\
\hline Qual a segunda gradua & & & & & & & & & & & & \\
\hline Área da saúde & 24 & 1 & 0 & & 20 & 5 & 0 & & 24 & 1 & 0 & \\
\hline Fora da área da saúde & 11 & 3 & 0 & 0.286 & 7 & 6 & 1 & 0.000 & 10 & 4 & 0 & 0.025 \\
\hline Não possui & 200 & 14 & 1 & & 158 & 57 & 0 & & 197 & 18 & 0 & \\
\hline
\end{tabular}

Nota: ${ }^{Z}$ Zona de conforto; ${ }^{2}$ Zona de alerta; ${ }^{3}$ Zona de Perigo. *CHI2. Fonte: Autores.

Quando avaliado os Fatores 1, 2 e 3 individualmente, a maioria das mulheres e dos homens encontravam-se na zona de conforto (ZC) para a colaboração interprofissional em todos os 3 fatores, tendo somente 2 alunas que se encontraram na zona de perigo (ZP) para TEC e IP. Isto quer dizer que a maior parcela dos entrevistados apresentou disposição para aprendizagem interprofissional, com forte significância estatística (p-valor $=0.005,0.016$ e 0.047 respectivamente para TEC, IP e ACP) $($ Tabela 3). 
Os estudantes do curso de graduação de Medicina, Enfermagem e Educação Física estavam, em sua maioria, na zona de conforto para TEC (p-valor $=0.048$ ), com somente 1 acadêmico na zona de perigo. Já os demais fatores não apresentaram significância estatística forte (IP e ACP com p-valor $=0.136$ e 0.260 respectivamente) (Tabela 3).

A grande maioria dos estudantes de primeira ou segunda graduação também se encontraram na ZC para colaboração interprofissional, com significância forte para IP (p-valor = 0.000) e ACP (p-valor=0.025) (Tabela 3).

Em geral, os estudantes tiveram uma disponibilidade para a aprendizagem interprofissional, encontrando-se em sua maioria, na zona de conforto, independentemente da variável avaliada.

\section{Discussão}

Os resultados encontrados por esta pesquisa permitem identificar que há uma alta disponibilidade dos estudantes dos cursos da saúde do Campus Pinheiro da UFMA para a aprendizagem interprofissional. Isto demonstra que a comunidade acadêmica reconhece a importância desse trabalho conjunto pois a colaboração interprofissional é uma competência que, quando trabalhada de maneira eficaz, irá contribuir para a melhoria do acesso aos serviços de saúde e melhora da qualidade da assistência a ser prestada; além de ter capacidade de melhorar as experiências dos pacientes e sua família no cuidado e o custo-efetividade da atenção em saúde (Peduzzi et al., 2020).

Apesar das estratégias formuladas nos últimos anos para superar o modelo de formação uniprofissional, o processo de formação dos cursos da área da saúde ainda é focado apenas nos conhecimentos específicos de cada profissão. Porém, reconhecendo esse cenário, não foi observado como um empecilho para os participantes apresentarem a disponibilidade para um novo modelo de formação focado no trabalho interprofissional dentro de uma equipe de saúde.

Da mesma forma que os resultados aqui observados, Barbosa (2020) indica que não houveram diferenças estatisticamente significativas quando consideradas as pontuações da escala quanto às variáveis sexo, idade e presença de graduação anterior. Neste estudo, bem como nos achados de Tompsen (2018), o que se observou foi uma concordância em não haver significância estatística na variável sexo. Estes estudos contrastaram com os resultados de Wilhelmsson (2011) que teve sua pesquisa realizada em duas Universidades suecas com estudantes dos cursos de Medicina e Enfermagem, e mostrou que acadêmicos em geral do sexo feminino e, especificamente do curso de Enfermagem, possuíam uma visão mais positiva da aprendizagem interprofissional, além de serem também mais abertos à colaboração com as demais profissões.

Em um estudo realizado por Nuto et al. (2017) com estudantes da UNIFOR que conta com cursos da área da saúde entre eles os três cursos participantes deste estudo: Medicina, Enfermagem e Educação Física, além dos cursos de Farmácia Fisioterapia, Fonoaudiologia, Odontologia, Nutrição, Psicologia e Terapia Ocupacional, apresentou resultados semelhantes aos observados nesta pesquisa. Segundo a pesquisa, os discentes que se encontravam em processo de conclusão de seus respectivos cursos apresentaram menor potencial para o desenvolvimento de competências colaborativas quando comparados aos discentes de níveis intermediários e ingressantes. Semelhante a este resultado, Barbosa (2020), demonstrou que há diferença significante quando se considera os estudantes em ciclos iniciais em relação ao fator 3 (ACP). Este resultado corrobora com os achados do presente trabalho pois evidenciou que estudantes de períodos intermediários apresentam maiores tendências ao trabalho em equipe.

O estudo realizado por Tompsen et al. (2018) com egressos dos cursos de Odontologia indicou também resultados animadores, revelando atitudes positivas dos participantes no que se refere ao trabalho em equipe, à colaboração interprofissional, à identidade profissional e, também, à atenção centrada no paciente, o que permite identificar um perfil inovador nos profissionais formados a partir desse novo modelo de formação com um olhar ampliado para a educação interprofissional e consequente trabalho interprofissional na prática profissional. 
Quando considera-se a atuação nos serviços de saúde, Vilela et al. (2018) conduziu estudos com profissionais de Medicina, Enfermagem, Fisioterapia, Psicologia e Nutrição, e revelou que, mesmo neste ambiente de prática fragmentada e uniprofissional e de formação influenciada ainda fortemente pelo modelo biomédico, observou-se que as competências relacionadas ao trabalho em equipe, à colaboração interprofissional e à atenção centrada no paciente obtiveram bons resultados. Porém, quando se avalia a competência de identidade profissional, observou-se no estudo que os valores se encontram em zona de alerta, assim como quando são analisados os valores por graduação.

Rodrigues (2018) realizou pesquisa com uma amostra estratificada em três grupos: Medicina, Enfermagem e demais profissões (Nutrição, Serviço Social, Odontologia, Psicologia, Farmácia, Fisioterapia e Biologia) e, diante disso, observou que profissionais da Medicina apresentam maior disponibilidade para o trabalho em equipe quando comparados aos demais profissionais em seu estudo, apesar de ainda assim os resultados obtidos estarem na zona de conforto, semelhante ao observado no estudo com alunos da UFMA - Pinheiro. Entretanto os dados foram claros quanto a IP e a baixa disponibilidade em reduzir as diferenças profissionais, tendo seus resultados em zona de alerta para as três categorias profissionais.

Num cenário pesquisado por Uchôa (2018) com participantes do estágio integrado em saúde, observou-se resultados semelhantes aos observados em nosso estudo com valores dentro da zona de conforto para TEC e ACP, porém observa-se que assim como no estudo de Rodrigues (2018) com profissionais, a IP também está em zona de alerta mesmo sem observar diferenças estatísticas relevantes.

O que se observa na ampla maioria dos estudos aqui citados, bem como nos resultados deste estudo, é que há uma alta disponibilidade dos estudantes, mas também de profissionais para a aprendizagem conjunta e integrada de saberes, podendo evidenciar um processo de mudanças nos cursos da saúde o que pode se dar em razão da influência de políticas em saúde que visam fomentar o trabalho interprofissional como o Pro-saúde e o PET - Saúde/Interprofissionalidade (Costa, 2015).

Ressalta-se ainda que, diferentemente de outras instituições que já disponibilizam aos seus estudantes a oportunidade de participar de disciplinas integradas com outros cursos da saúde que apesentaram resultados satisfatórios, observa-se que ainda não há na Universidade Federal do Maranhão - Campus Pinheiro essa opção em suas grades curriculares, apesar das propostas iniciais de reformulação dos projetos pedagógicos dos três cursos da área da saúde do campus. Porém, apesar dessa diferença importante, o que se observou foram resultados semelhantes com a ampla maioria dos estudos aqui citados.

Apenas num cenário onde a formação centrada em habilidades e no perfil específico das categorias profissionais e da clara divisão do trabalho em saúde forem superados, a aprendizagem interprofissional e o trabalho colaborativo será possível, rompendo com as estruturas tradicionais centradas apenas em disciplinas. Determinantes históricos, sociais, econômicos e culturais exigem transformações no perfil dos profissionais que vem sendo formado pelas instituições, para que sejam implementadas formas eficientes e resolutivas no fazer saúde (Rossit et al., 2018).

É importante destacar que o trabalho apresentou algumas limitações. Em primeiro lugar, ressalta-se que a amostra é por conveniência, desse modo, o resultado não pode ser generalizado, limitando-se aos discentes da unidade em que ocorreu a coleta de dados. Outro aspecto que deve ser considerado é o p-valor, o qual não mostrou grande significância estatística quando se considerou a média dos fatores da escala RIPLS segundo as características sociodemográficas e acadêmicas (Tabela 2). Assim, não foi possível estabelecer correlação entre tais características e os fatores da interprofissionalidade, ainda que algumas variáveis tenham se mostrado com maior predisposição estatisticamente significativa para zona de conforto em relação aos fatores.

Esses dados contribuem com informações relevantes para o planejamento da Educação Interprofissional (EIP) no ambiente universitário nas áreas da saúde ao passo que apresentam características dos estudantes, tais como período da graduação, curso e graduações anteriores, que demonstram maior disponibilidade ou não de se engajar na EIP. Ademais, este estudo acrescenta dados estatísticos importantes à literatura na língua portuguesa sobre a interprofissionalidade, a qual ainda é pouco desenvolvida. 


\section{Conclusão}

Sob a perspectiva dos alunos de graduação dos cursos de Medicina, Enfermagem e Educação Física acerca da EIP e práticas colaborativas, o presente estudo concluiu com resultado satisfatório que os alunos durante seu processo de formação profissional possuem aptidão e disponibilidade com alta significância para aceitarem propostas pedagógicas que abordem a prática interprofissional. Desta forma, a hipótese proposta pelo estudo, na qual se descreve que a EIP é conivente e aceitável ao ser inserida no processo de formação acadêmica, foi aceita.

Além do mais, os resultados obtidos respeitaram o quantitativo amostral necessário e de relevância científica significativa. Logo, os resultados obtidos de 254 questionários que consideraram os fatores socioeconômicos, de idade, ou o fator de estar cursando uma segunda graduação, demonstraram-se estatisticamente significativos ao serem relacionados à EIP e às práticas colaborativas, mostrando assim que grande maioria dos pesquisados se encontram em Zona de Conforto (ZC) em relação a Educação Interprofissional e Práticas Colaborativas no ambiente acadêmico.

Inteira-se, que o estudo apresenta a adesão da EIP e práticas colaborativas como adequada a todos os períodos da graduação, sendo mais aceita nos períodos clínicos/fase intermediária dos cursos, onde se sugere que as práticas colaborativas serão mais exigidas. Ademais, o estudo destaca que atividades extracurriculares, como projetos e programas de ensino, pesquisa e extensão são ferramentas indispensáveis no processo de ensino-aprendizagem da EIP e práticas colaborativas.

Cabe ressaltar que é totalmente necessário a criação e manutenção de ambientes que abordem a discussão da EIP, tendo como objetivo aprofundar conhecimentos e inserir as práticas colaborativas nas instituições de ensino e de prática assistencial.

Com isso, estudos com diferentes variáveis e em campos expandidos como no setor assistencial de saúde se fazem necessários para uma compreensão mais abrangente acerca da temática, objetivando entender os efeitos positivos e os desafios encontrados na execução da prática interprofissional.

\section{Referencias}

Amado, E., \& Vilela, R. B. (2017). Educação interprofissional e prática colaborativa em terapia intensiva: perspectiva dos preceptores. Encontro Internacional de Formação de Professores e Fórum Permanente de Inovação Educacional, 10(1).

Barbosa, G. R. (2020). Disponibilidade de estudantes de oito cursos da saúde de uma universidade pública brasileira para a educação interprofissional (Tese de doutorado). Universidade Estadual de Campinas, Faculdade de Ciências Médicas. http://repositorio.unicamp.br/bitstream/REPOSIP/346070 /1/Barbosa_GuilhermeRodrigues_D.pdf

Barros, E. R., \& Ellery, A. E. L. (2016). Colaboração interprofissional em uma unidade de terapia intensiva: desafios e possibilidades. Revista da Rede de Enfermagem do Nordeste, 17(1), 10-19.

Casanova, I. A., Batista, N. A., \& Moreno, L. R. (2018). A Educação Interprofissional e a prática compartilhada em programas de residência multiprofissional em Saúde. Interface-Comunicação, Saúde, Educação, 22, 1325-1337.

Costa, M. V. D. (2016). A educação interprofissional no contexto brasileiro: algumas reflexões. Interface-Comunicação, Saúde, Educação, 20 , $197-198$.

Costa, M. V. D., \& Borges, F. A. (2015). O Pró-PET-Saúde frente aos desafios do processo de formação profissional em saúde. Interface-Comunicação, Saúde, Educação, 19, 753-763.

Crisp, N., \& Chen, L. (2014). Global supply of health professionals. New England Journal of Medicine, 370(10), 950-957.

Frenk, J., Chen, L., Bhutta, Z. A., Cohen, J., Crisp, N., Evans, T., \& Zurayk, H. (2010). Health professionals for a new century: transforming education to strengthen health systems in an interdependent world. The lancet, 376(9756), 1923-1958.

Gallotti, F. C. M., Dos Santos, L. E. R., Dias, V. G. A., dos Santos Farias, Q. S., Martins, M. D. C. V., de Góis, R. M. O., \& Serafini, M. R. (2021). Formação do enfermeiro na perspectiva do cuidado integral e trabalho em equipe. Research, Society and Development, 10(1), e24110111724-e24110111724.

Haugland, M., Brenna, S. J., \& Aanes, M. M. (2019). Interprofessional education as a contributor to professional and interprofessional identities. Journal of interprofessional care, 1-7.

Institute of Medicine (2015). Measuring the Impact of Interprofessional Education on Collaborative Practice and Patient Outcomes. The National Academies Press.

Nuto, S. D. A. S., Lima Júnior, F. C. M., Camara, A. M. C. S., \& Gonçalves, C. B. C. (2017). Avaliação da disponibilidade para aprendizagem interprofissional de estudantes de Ciências da Saúde. Revista Brasileira de Educação Médica, 41(1), 50-57.

Organização Mundial da Saúde. (2010). Marco para ação em educação interprofissional e prática colaborativa. Editora Freelance. 
Research, Society and Development, v. 10, n. 5, e5110514743, 2021

(CC BY 4.0) | ISSN 2525-3409 | DOI: http://dx.doi.org/10.33448/rsd-v10i4.14743

Peduzzi, M., Agreli, H. L. F., Silva, J. A. M. D., \& Souza, H. S. D. (2020). Trabalho em equipe: uma revisita ao conceito e a seus desdobramentos no trabalho interprofissional. Trabalho, Educação e Saúde, 18.

Reeves, S., Lewin, S., Espin, S., \& Zwarenstein, M. (2010). Interprofessional teamwork for health and social care. Oxford, UK: Blackwell-Wiley. [Crossref], [Google Scholar].

Reeves, S., Xyrichis, A., \& Zwarenstein, M. (2017). Teamwork, collaboration, coordination, and networking: Why we need to distinguish between different types of interprofessional practice. Journal of Interprofessional Care, 32(1), 1-3. https://doi.org/10.1080/13561820.2017.1400150

Rodrigues, J.R.S. (2018). Educação interprofissional em um hospital de trauma no agreste alagoano: perspectivas dos profissionais do NEP em saúde e seus colaboradores (Dissertação de Mestrado). Faculdade de Medicina, Programa de Pós-Graduação em Ensino na Saúde. Universidade Federal de Alagoas.

Rossit, R. A. S., Freitas, M. A. D. O., Batista, S. H. S. D. S., \& Batista, N. A. (2018). Construção da identidade profissional na educação interprofissional em saúde: percepção de egressos. Interface-Comunicação, Saúde, Educação, 22, 1399-1410.

Souto, T. D. S., Batista, S. H., \& Alves Batista, N. (2014). A educação interprofissional na formação em psicologia: olhares de estudantes. Psicologia: ciência e profissão, 34(1), 32-45.

Tompsen, N. N., MEIRELES, E., PEDUZZI, M., \& TOASSI, R. F. C. (2018). Educação interprofissional na graduação em Odontologia: experiências curriculares e disponibilidade de estudantes. Revista de Odontologia da UNESP, 47(5), 309-320.

Uchôa, P. d. A. (2018). Estágio integrado em Saúde e aprendizagem interprofissional: percepção dos discentes [Dissertação de Mestrado, Faculdade de Medicina, Programa de Pós Graduação em Ensino na Saúde, Universidade Federal de Alagoas]. http://www.repositorio.ufal.br/handle/riufal/3409

Vilela, R. Q. B., \& Amado, E. (2018). Educação interprofissional e prática colaborativa em terapia intensiva: perspectiva dos profissionais de saúde. Brazilian Applied Science Review, 2(4), 1247-1268.

Wilhelmsson, M., Ponzer, S., Dahlgren, L. O., Timpka, T., \& Faresjö, T. (2011). Are female students in general and nursing students more ready for teamwork and interprofessional collaboration in healthcare?. BMC medical education, 11(1), 1-10. 